\title{
Newly referred patients engaged in an ongoing and uncertain process to understand the causes of their psychological problems
}

Williams B, Healy D. Perceptions of illness causation among new referrals to a community mental health team: "explanatory model"or "exploratory map". Soc Sci Med 2001 Aug;53:465-76.

QUESTION: What are the perceptions of patients newly referred to a community mental health team (CMHT) about the causes of their psychological experiences?

Design

Grounded theory.

\section{Setting}

Rural North Wales, UK.

\section{Participants}

15 patients with no known history of mental health problems who were new referrals to a CMHT. None had psychotic symptoms or were later given a diagnosis of psychosis.

\section{Methods}

Patients participated in unstructured, home interviews before their first appointment and approximately 2 weeks after the appointment. They were asked to explain how they had arrived at their current situation. Interviews were transcribed and analysed using constant comparison methods. Validity of findings was checked in second interviews (after the first appointment), and through 8 additional interviews with individuals who were currently in contact with the CMHT or who had ceased contact within the previous 6 months.

\section{Main findings}

Most patients did not have definitive explanations about the cause of their current problems. Some reported several possible causative events or occurrences; others cited one explanation, only to cite an alternative, sometimes contradictory, explanation later in the interview. Patients expressed an explicit or implicit lack of certainty about the "true" cause. They sought explanations, with varying levels of success, and struggled with the social implications of these explanations.

The concept of an "exploratory map" was used to represent the various avenues of thought that patients explored as they tried to understand the reasons for their current psychological problems. The range of possible causes could be classified along 2 dimensions: origin (social or biological) and relation to the self (external, internal, or direct). The importance of the concept and role of self became apparent in situations where patients felt that the severity of the psychological experience was disproportionate to the severity of the perceived causes. Causes were seen to be mediated or processed by some concept, characterised as "strength", which was defined as the "ability to resist or recover from external events of either social or biological origin".

Most patients attributed their psychological experiences to multiple external causes of varying severity. Some events were expected to lead to a greater severity of psychological experience than others. External social causes included life events such as the death of friends or family or a difficult work environment. External biological causes included, for example, sudden injuries or chronic illness. Internal causes tended only to be social in origin, and often included past traumatic events that had done some internal or latent damage to the mind/ self (eg, childhood abuse). Direct causes, biological in origin, were seen to "bypass" the mind/self and directly influence the psychological experience. Examples included premenstrual syndrome and postnatal depression. Because the mind/self was bypassed, the resulting psychological experience was not seen as a reflection of an individual's strength.

\section{Conclusions}

People facing a mental health problem for the first time engaged in an ongoing and uncertain process of trying to make sense of and seek meaning in their psychological experience. Perceived causes of problems were conceptualised along the dimensions of origin (social or biological) and relation to the self (internal, external, or direct)
Source of funding: not
stated.

For correspondence: Dr B Williams, Department of Epidemiology and Public Health, Ninewells Hospital and Medical School, Dundee, UK.

bwilliams@ eph.dundee.ac.uk

\section{COMMENTARY}

The purpose of this study by Williams and Healy was to explore patients' views on why they sought help from a community mental health team. The rigour of the study was enhanced by a second researcher independently scrutinising the data collected and by theoretical sampling until saturation of emergent categories occurred.

The findings show that people want to make sense of their psychological experiences and discover their causes. Participants identified more than 1 reason for their current psychological experiences. These reasons were categorised into social or biological explanations and/or explanations related to self (external, internal, or direct). This left me wondering if there could be other reasons, as all seem to be covered within this framework.

A dynamic, fluid exploratory map represented the core category that emerged and suggested several implications for practice. The map could be used to depict the changing nature of human beings and how meanings can change and suggests that patients need time to search for reasons for their experiences. Clinicians need to be aware that these perceived reasons might change with time, in both the strength of their meaning and the length of time they affect individuals. Indeed, findings showed that $>2$ reasons might be given and, at times, these reasons might even contradict each other. However, movement and uncertainty are not in themselves always confusing. At times, patients may need to rely on one explanation, which seems more uncomplicated than others, to avoid further pain and preserve hope. Clinicians need to provide true, patient centred care and be aware that patients might need to explore various explanations. This type of care begins the journey of healing from within patients' frames of reference (ie, their own beliefs, thoughts, and feelings). It continues by exploring patients' personal pathways on their own exploratory maps (which they could choose to talk about, write about, draw, or paint) throughout the total care process. The use of maps requires further research.

Ann Long, RGN, RMN, HV, MSc, DPhil University of Ulster at Jordanstown Newtownabbey, North Ireland 